hep-th/0010060

EFI-00-38

\title{
Exact Noncommutative Solitons
}

\author{
Jeffrey A. Harvey, Per Kraus, and Finn Larsen \\ Enrico Fermi Institute and Department of Physics \\ University of Chicago, \\ 5640 S. Ellis Ave., Chicago, IL 60637, USA
}

\begin{abstract}
We find exact solitons in a large class of noncommutative gauge theories using a simple solution generating technique. The solitons in the effective field theory description of open string field theory are interpreted as D-branes for any value of the noncommutativity. We discuss the vacuum structure of open string field theory in view of our results.
\end{abstract}

October, 2000 


\section{Introduction}

The study of semi-classical solutions of noncommutative field theories has turned out to be an interesting and rich subject with a variety of unexpected applications. The properties of noncommutative $U(1)$ instantons provided one of the early motivations for the study of noncommutative field theories [1], and it has been understood that noncommutative gauge theory arises from a limit of string theory [2, 3, [1, 5]. More recently soliton solutions of scalar noncommutative field theory have been constructed [6]. These solutions have played an important role in constructing D-branes as noncommutative solitons of the tachyon field of open string theory [7, 8, 9]. There have also been many studies of solitons in noncommutative scalar-gauge theory $[8,10,11,12,13,14,15,16,17]$.

In this paper we introduce a simple technique to find exact solutions in many different noncommutative gauge field theories, with or without scalar fields. We exploit it to generate several new solutions, as well as some that were previously presented in the literature. Our method provides an efficient means of finding non-trivial solutions, and additionally offers a unified interpretation of a large class of solitons.

The starting point of our solution generating technique is the gauge invariance of noncommutative gauge theory. We then note that an enlarged class of transformations leave the field equations invariant by virtue of being "almost gauge", without being full-fledged symmetries. These transformations thus map solutions to solutions, giving a simple method to generate new solutions. In this paper we describe the method for gauge field theories and give various examples, including a detailed discussion of the effective field theory description of open string field theory. We anticipate that generalizations of the construction can be applied directly in the full string field theory, both in its cubic formulation [18], and its background independent version [19].

The solitons we construct are exact solutions for all values of the noncommutativity parameter, or equivalently the background $B$-field. They are regular for any non-vanishing value of $B$, but singular in the limit $B \rightarrow 0$. They should therefore be distinguished from solutions which remain smooth as $B \rightarrow 0$; those form a seperate branch and are more difficult to construct explicitly. For example, some of the familiar BPS solitons in commutative theories have generalizations to the noncommutative case [12] but the solitons we construct typically have higher energy than the corresponding BPS solitons; indeed we find that in some cases their energy diverges as $B \rightarrow 0$.

An important application of noncommutative solitons is to the description of tachyon 
condensation in open string field theory. Large noncommutativity $B=\infty$ introduces a large length scale making derivatives in the action negligible; so in this limit it is simple to find $p$-brane solitons. Explicit computation shows that they have the same tension and other properties as $D$-branes; they should therefore be identified with $D$-branes [8]. In this paper we generalize this result by exhibiting solitons with the same tension as $D$-branes for all values of $B$. The new feature in the present construction is that there is a nonvanishing gauge field excited in the solution, adjusted precisely so that the gauge covariant derivatives vanish identically. This property effectively makes derivatives unimportant without needing a new length scale to justify neglecting them. Moreover, the form of the string field theory effective action is such that this feat can be accomplished at no cost in energy.

In our description D-branes are thus interpreted as solitons, with a width that depends on $B$ and vanishes as $B \rightarrow 0$. This result is satisfying because $D$-branes are smaller than string scale in perturbative string theory at $B=0$. If the aim is to understand the solitons at $B=0$ it may be useful to interpret the noncommutativity as a regulator which can be taken arbitrarily small. The physical picture is qualitatively similar to the one emerging from boundary string field theory [20,21] (see also [22]). There the boundary conformal field theory is perturbed by a relevant operator with the mass parameter $u$. The soliton has width $1 / u$ and $u$ flows to $u=\infty$ in the exact description; thus there is a rough correspondance $B \leftrightarrow 1 / u$. In contrast, the description of D-brane solitons in the level truncation approximation to the cubic string field theory appears to yield solitons of finite width [23]. The field variables used in our discussion are evidently qualitatively similar to those of boundary string field theory, but not those of the level truncation scheme.

One of the goals of studying tachyon condensation in open string field theory is to obtain a better understanding of the non-perturbative "closed string" vacuum and its symmetries. In open string theory there is an obvious gauge symmetry changing $F$ and $B$ while fixing $\mathcal{F}=F+B$. Thus the gauge invariant characterization of large noncommutativity is $\mathcal{F}=\infty$. In the closed string vacuum far away from any $\mathrm{D}$-branes there are no gauge fields to give rise to $F$, and different constant values of $B$ are gauge equivalent. This gauge invariance is quite mysterious from the open string point of view. Our results give a direct calculational verification of the expected $\mathcal{F}$ independence of $D$-brane properties. The relation of our results to other proposals regarding the structure of the closed string vacuum will be discussed in the final section of this paper. 
The paper is organized as follows. In section 2 we present the solution generating technique for noncommutative solitons and give examples in simple noncommutative gauge theories. In section 3 we use an effective field theory description of open string field theory to find solitons that we interpret as $D$-branes for any value of $\mathcal{F}$. Section 4 discusses some implications of our results.

We note that several papers have exploited partial isometries in the construction of instanton solutions [24,25,26] and in a study of string field theory [27].

\section{The Solution Generating Technique}

In many physical theories the equations of motion are left invariant by a larger symmetry than is the full theory. This property is useful for generating nontrivial solutions to the field equations: starting with a known (and typically simple) solution and acting with a symmetry of the equations of motion one finds a new (and often more involved) solution. In the present section we apply this strategy to noncommutative gauge theories and consider as examples Yang-Mills-Higgs theories. The following section discusses the effective theories arising in open string field theory. In both cases, we will start with the vacuum and act with a solution generating transformation to arrive at a soliton solution. The solutions we construct are exact for any value of noncommutativity. One class of these solutions reduces to those of [6] in the limit of large noncommutativity, while another class gives exact vortex solutions and higher dimensional generalizations thereof.

\subsection{The General Construction}

A quantum field theory in a given dimension can always be represented as a lower dimensional theory, with the "missing" dimensions implemented as additional indices on the fields. This type of representation arises naturally in D-brane realizations of noncommutative field theories. Here D-branes can be represented as configurations of infinitely many lower dimensional D-branes. In this framework, the familiar $U(1)$ gauge theory on the brane becomes a " $U(\infty)$ " gauge symmetry in the lower dimensional theory and implements the invariance under area-preserving diffeomorphisms [28,29].

It is convenient to represent field configurations on the noncommutative space as operators acting on an auxiliary Hilbert space $\mathcal{H}$ (see e.g. [6].) In this formalism the symmetry is more precisely written as a $U(\mathcal{H})$ symmetry of unitary transformations on 
Hilbert space transforming states and operators as

$$
\begin{aligned}
|\psi\rangle & \rightarrow U|\psi\rangle, \\
\langle\psi| & \rightarrow\langle\psi| \bar{U}, \\
\mathcal{O} & \rightarrow U \mathcal{O} \bar{U} .
\end{aligned}
$$

where $\overline{\mathcal{O}}$ denotes the Hermitian conjugate or adjoint of $\mathcal{O}$. In the operator representation the equations of motion can be arranged to involve products of operators each transforming according to the last equation in (2.1). They are clearly invariant under transformations satisfying

$$
\bar{U} U=I
$$

That is, the condition (2.2) ensures that

$$
\frac{\delta S}{\delta \mathcal{O}} \rightarrow U \frac{\delta S}{\delta \mathcal{O}} \bar{U}
$$

and so will take solutions of the equations of motion to solutions.

True gauge transformations of the theory are realized by unitary operators satisfying $U \bar{U}=I$ as well as (2.2). This should be distinguished from the present situation where the solution generating transformations are not full-fledged symmetries of the theory; in particular, they do not leave the action invariant. The condition (2.2) implies that $U \bar{U}$ is a projection operator, so solution generating transformations are represented by the operators satisfying (2.2) but

$$
U \bar{U}=P
$$

with $P$ a projection operator not equal to the identity operator.

The operator equation $O \bar{O} O=O$ implies that $O \bar{O}=P_{1}$ and $\bar{O} O=P_{2}$ where $P_{1}, P_{2}$ are projection operators. Projection operators related in this way are said to be MurrayVon Neumann equivalent. An operator obeying this equation is called a partial isometry. If $P_{2}$ is the identity operator then $O$ preserves inner products on the full Hilbert space and is thus called an isometry. If $P_{1}$ is also the identity operator then $O$ is unitary. Thus our generating transformations $U$ are non-unitary isometries and $P$ is Murray-von Neumann equivalent to the identity operator.

The solution generating transformations are "almost" gauge transformations. For $P=I-P_{n}$, with $P_{n}$ a rank $n$ projection operator, $U$ fails to be unitary only in an $n$ dimensional subspace. In position space, the corresponding statement is that $U$ fails to be 
a true gauge transformation in a region of characteristic size $\sqrt{n \theta}$ around the origin. Thus acting with $U$ on the vacuum will produce localized soliton solutions. Since $U$ does not generally commute with the Hamiltonian, the solitons will have nonvanishing energy. These transformations should not be confused with more familiar "large" gauge transformations. The latter leave the classical action invariant but act nontrivially on quantum states due to their nontrivial behavior at infinity.

It is often the case that the full Hilbert space factorizes into several subspaces, e.g. corresponding to pairs of noncommutative dimensions, or commutative ones. A more general solution generator can then be found which acts as a non-unitary isometry on each subspace independently. Apart from the obvious interest in generalizing the construction, this is useful because we are often interested in solutions which do not depend on time, or other variables "along the brane". Such solutions are generated by choosing the identity transformation along the appropriate subspaces. More generally, we can take the transformations to act by taking states from one subspace into those of another.

It is not possible to realize (2.2) and (2.4) simultaneously in a finite dimensional vector space. To construct examples in an infinite dimensional separable Hilbert space, introduce an orthonormal basis $|k\rangle, k=0,1, \ldots$ and consider the shift operator

$$
S=\sum_{k=0}^{\infty}|k+1\rangle\langle k| .
$$

It satisfies

$$
\bar{S}^{n} S^{n}=I, \quad S^{n} \bar{S}^{n}=I-P_{n}
$$

where $I$ is the identity operator and $P_{n}$ denote the projection operators on to the first $n$ states

$$
P_{n}=\sum_{k=0}^{n-1}|k\rangle\langle k| .
$$

Thus $U=S^{n}$ are solution generating transformations.

In the remainder of this section we show how these abstract considerations work in some explicit examples. We will need the following notation. Coordinates commute according to

$$
\left[x^{i}, x^{j}\right]=i \Theta^{i j} .
$$

In $2+1$ dimensions we write $\theta=\Theta^{12}$. In higher dimensions we skew-diagonalize $\Theta$ with $\theta^{i}=\Theta^{i, i+1}$. Integrals over any two noncommutative directions appear in the operator 
representation as

$$
\frac{1}{2 \pi \theta} \int d^{2} x \rightarrow \operatorname{Tr}
$$

In complex coordinates, $z=\left(x^{1}+i x^{2}\right) / \sqrt{2}$, derivatives become

$$
\partial=\partial_{z}=-\theta^{-\frac{1}{2}}[\bar{a}, \cdot], \quad \bar{\partial}=\partial_{\bar{z}}=\theta^{-\frac{1}{2}}[a, \cdot],
$$

where $a=z / \sqrt{\theta}$ so that

$$
[a, \bar{a}]=I
$$

We write the gauge potential and field strength as

$$
\begin{aligned}
& A=A_{z}, \quad \bar{A}=A_{\bar{z}} \\
& F=i F_{z \bar{z}}=i(\partial \bar{A}-\bar{\partial} A-i[A, \bar{A}])=\theta^{-1}([C, \bar{C}]+I),
\end{aligned}
$$

where

$$
C=\bar{a}+i \theta^{\frac{1}{2}} A, \quad \bar{C}=a-i \theta^{\frac{1}{2}} \bar{A} .
$$

When considering noncommutativity in several complex dimensions simultaneously an additional index is introduced on the various symbols to distinguish subspaces.

\subsection{Soliton Examples}

Consider a noncommutative gauge theory in $D=2+1$ dimensions

$$
S=\int d t d^{2} x\left(-\frac{1}{4}\left(F_{\mu \nu}\right)^{2}+\frac{1}{2} D^{\mu} \phi D_{\mu} \phi-V\left(\phi-\phi_{\star}\right)\right) .
$$

Our metric convention is $g_{\mu \nu}=(+,-,-)$. We take the potential $V$ to have a local minimum at $\phi=\phi_{*}$ with $V(0)=0$ and a local maximum at $\phi=0$. We take the scalar field in the adjoint representation so the covariant derivative is

$$
D_{\mu} \phi=\partial_{\mu} \phi-i\left[A_{\mu}, \phi\right]
$$

or in the operator formalism

$$
\begin{aligned}
& D \phi \equiv D_{z} \phi=-\theta^{-\frac{1}{2}}[C, \phi], \\
& \bar{D} \phi \equiv D_{\bar{z}} \phi=\theta^{-\frac{1}{2}}[\bar{C}, \phi] .
\end{aligned}
$$

The simplest solutions to the theory (2.14) are spatially uniform, with the scalar field equal to an extremum of the potential, $\phi=\phi_{\star}$, and the gauge field vanishing, i.e. $C=\bar{a}$ and $\bar{C}=$ 
a. In (2.14) the scalar field has been shifted such that the Lagrangian has no terms linear in the fields. This is important because otherwise the equations of motion would have a term proportional to the identity operator $I$ which transforms in the identity representation (for non-unitary transformations the adjoint representation would take $I \rightarrow U I \bar{U}=P$ ). Thus each term in the equations of motion transforms in the adjoint of $U(\mathcal{H})$ so, without detailed computations it is clear that they are invariant under "gauge" transformations $\phi \rightarrow U \phi \bar{U}, C \rightarrow U C \bar{U}$ even for non-unitary $U$ satisfying (2.2) and (2.4). Taking $U=S^{n}$ and using (2.6) we generate the soliton solutions

$$
\begin{aligned}
\phi & =\phi_{\star}\left(I-P_{n}\right), \\
C & =S^{n} \bar{a} \bar{S}^{n}, \\
\bar{C} & =S^{n} a \bar{S}^{n} .
\end{aligned}
$$

Note that here and in all other solutions considered in this paper the barred gauge field $\bar{C}$ is simply the hermitian conjugate of $C$.

In position space the operator $P_{n}$ vanishes exponentially outside a region of linear extent $\sim \sqrt{n \theta}$; so the tachyon field in (2.17) is excited from the vacuum $\phi=\phi_{\star} I$ in a region of this size. The field strength

$$
F=\frac{1}{\theta}([C, \bar{C}]+I)=\frac{1}{\theta} P_{n}
$$

is similarly localized, so $C=\bar{a}$ asymptotically. The solution (2.17) is thus interpreted as a well-localized soliton.

In operator form the energy of static solutions to the theory (2.14) is

$$
E=2 \pi \theta \operatorname{Tr}\left(\frac{1}{2} F^{2}+\frac{1}{\theta}[C, \phi][\bar{C}, \phi]+V\left(\phi-\phi_{\star}\right)\right)
$$

We normalize the potential so that the state far from the soliton has vanishing energy $V(0)=0$ (otherwise the energy would diverge). Then the solution (2.17) has energy

$$
E=2 \pi \theta n\left(\frac{1}{2 \theta^{2}}+V\left(-\phi_{\star}\right)\right)
$$

As far as we are aware the soliton solutions (2.17) are new. At infinite noncommutativity the gauge field is negligible and our solutions reduce to the pure scalar field solutions of [6]. For $\phi_{\star}=0$ they reduce to the pure gauge theory solutions found in [11] and studied 
in detail in 16]. Note that the energy (2.20) diverges as $\theta \rightarrow 0$, so we do not find well behaved solutions in the commutative theory.

A variation of the construction above is to reconsider the theory (2.14) but taking instead the scalar field $\phi$ in the fundamental representation of the gauge group. Then $\phi \rightarrow U \phi$ under gauge transformations. Following the steps above we find solutions with

$$
\phi=S^{n} \phi_{\star}
$$

and the gauge fields given in (2.17). For a quartic potential, this solution was first found in [15] and represents a vortex. Generalizations are considered in the following section.

\subsection{Vortex Examples}

Motivated by brane constructions it is natural to consider theories with a complex scalar field $\phi$ transforming in the bi-fundamental of two $U(1)$ gauge groups. The Lagrangian

$$
S=\int\left(-\frac{1}{4}\left(F_{\mu \nu}^{+}\right)^{2}-\frac{1}{4}\left(F_{\mu \nu}^{-}\right)^{2}+\frac{1}{4}\left(D^{\mu} \phi D_{\mu} \bar{\phi}+D^{\mu} \bar{\phi} D_{\mu} \phi\right)+V(\phi \bar{\phi}-1)+W(\bar{\phi} \phi-1)\right)
$$

with covariant derivatives

$$
\begin{aligned}
& D_{\mu} \phi=\partial_{\mu} \phi-i\left(A_{\mu}^{+} \phi-\phi A_{\mu}^{-}\right) \\
& D_{\mu} \bar{\phi}=\partial_{\mu} \bar{\phi}+i\left(\bar{\phi} A_{\mu}^{+}-A_{\mu}^{-\bar{\phi}}\right)
\end{aligned}
$$

is invariant under the $U(\mathcal{H}) \otimes U(\mathcal{H})$ gauge symmetry

$$
\begin{gathered}
\phi \rightarrow V \phi \bar{U}, \\
C^{-} \rightarrow U C^{-} \bar{U}, \\
C^{+} \rightarrow V C^{+} \bar{V} .
\end{gathered}
$$

The non-unitary isometries $U=S^{n}, V=S^{m}$ generate the solutions

$$
\begin{aligned}
\phi & =S^{m} \bar{S}^{n}, \\
C^{-} & =S^{n} \bar{a} \bar{S}^{n}, \\
C^{+} & =S^{m} \bar{a}^{m} .
\end{aligned}
$$

The corresponding field strengths

$$
F^{-}=\frac{1}{\theta} P_{n}, \quad F^{+}=\frac{1}{\theta} P_{m}
$$


identify the solutions (2.25) as coincident vortices, charged with respect to both $U(1)$ fields. The flux is quantized,

$$
\int d^{2} x F^{ \pm}=2 \pi \theta \operatorname{Tr}\left(F^{ \pm}\right)=2 \pi \text { (integer) }
$$

In string theory these solutions will be interpreted as vortex/anti-vortex configurations corresponding to coincident branes and anti-branes.

It can be verified by explicit computation that 2.25) satisfy the equations of motion. A first step is the operator expressions

$$
\begin{aligned}
& D \phi=\theta^{-\frac{1}{2}}\left(-C^{+} \phi+\phi C^{-}\right), \\
& D \bar{\phi}=\theta^{-\frac{1}{2}}\left(-C^{-} \bar{\phi}+\bar{\phi} C^{+}\right),
\end{aligned}
$$

giving

$$
D \phi=D \bar{\phi}=\bar{D} \phi=\overline{D \phi}=0 \text {. }
$$

These relations are not surprising because they hold trivially in vacuum, and they are preserved by the solution generating transformation.

In the limit of large noncommutativity a class of these solutions was first found in [8,9]. Our solutions are singular in the $\theta \rightarrow 0$ limit. This is to be contrasted with the approximate vortex solutions studied in [12]. The latter reduce to the standard Nielsen-Olesen vortex in the commutative limit.

\subsection{The ABS Construction}

The vortex solution can be generalized to solitons localized in more dimensions. The relevant construction is due to Atiyah-Bott-Shapiro (ABS) [30] and is well known from the construction of BPS solitons in the $D \bar{D}$-system [31]. Our discussion follows the noncommutative generalization of these solutions in [32] where more details can be found (see also [31,9).

We want to construct a soliton of co-dimension $2 p$. The starting point is a set of gamma matrices $\Gamma_{j}, j=1,2, \ldots 2 p$ which map $S_{+}$to $S_{-}$where $S_{ \pm}$are the two $2^{p-1}$ dimensional spinor representations of the $S O(2 p)$ rotation group transverse to the soliton. General principles give [32]

$$
\begin{aligned}
& \operatorname{dim} \operatorname{ker} \Gamma \cdot x=1, \\
& \operatorname{dim} \operatorname{ker} \bar{\Gamma} \cdot x=0,
\end{aligned}
$$


with suitable conventions for $\Gamma$-matrices. It follows that

$$
\bar{T}=\bar{\Gamma} \cdot x \frac{1}{\sqrt{\Gamma \cdot x \bar{\Gamma} \cdot x}}
$$

is well defined and satisfies

$$
T \bar{T}=I, \quad \bar{T} T=I-P_{1},
$$

where $P_{1}$ is the projection operator onto the one-dimensional kernel of $\Gamma \cdot x$. It is now clear that $U=\bar{T}$ is a solution generating transformation. The resulting gauge fields are

$$
C_{\alpha}^{+}=\bar{a}_{\alpha}, \quad C_{\alpha}^{-}=\bar{T} \bar{a}_{\alpha} T
$$

where the index $\alpha=1, \ldots, p$ ennumerates the subspaces.

Let us be more explicit for the case of $p=2$. We label the two 2D subspaces by coordinates $z$ and $w$, and work in terms of operators acting on the Hilbert space of two by two matrices with entries in $\mathcal{H} \otimes \mathcal{H}$. The gauge fields (2.33) then become

$$
C_{z}^{+}=\bar{a} \otimes I, \quad C_{w}^{+}=I \otimes \bar{a}, \quad C_{z}^{-}=\bar{T}(\bar{a} \otimes I) T, \quad C_{w}^{-}=\bar{T}(I \otimes \bar{a}) T .
$$

Choosing the noncommutativity $\theta$ in the two subspaces to be equal, the corresponding nonvanishing field strengths are

$$
F_{z \bar{z}}^{-}=F_{w \bar{w}}^{-}=-i \theta^{-1} P_{1}
$$

This is a self-dual field strength (see below).

The construction of multi-vortex/anti-vortex solutions from the previous section can be repeated in $2 p$ dimensions. The operator $T$ has a one-dimensional kernel and the first equation in (2.32) implies that it is surjective, $\operatorname{im} T=S_{-} \otimes \mathcal{H}^{\otimes p}$. It follows that $T$ maps a one-dimensional subspace into its own kernel, a process that can be repeated with the result (this also follows from an index theorem [33] )

$$
\operatorname{dim} \operatorname{ker} T^{n}=n,
$$

and so

$$
T^{n} \bar{T}^{n}=I, \quad \bar{T}^{n} T^{n}=I-P_{n},
$$


where $P_{n}$ is the projection operator onto the kernel of $T^{n}$. We can thus use the operator $U=\bar{T}^{n}$ as a solution generator; moreover there are two gauge fields transforming independently, as in (2.24), so we can also use $V=\bar{T}^{m}$. The result is multi-ABS/anti-ABS solitons with the gauge fields

$$
C_{\alpha}^{+}=\bar{T}^{m} \bar{a}_{\alpha} T^{m}, \quad C_{\alpha}^{-}=\bar{T}^{n} \bar{a}_{\alpha} T^{n}
$$

We have several comments:

(1) The manipulations above are quite general; they can be repeated after replacing $\Gamma \cdot x$ with any other operator satisfying (2.30). The operators $T$ and $\bar{T}$ are known as Toeplitz operators.

(2) The derivation illustrates a general feature of the solution generating technique, namely that we do not need an explicit action to find a solution, only some general properties of the theory.

(3) The gauge field $C^{-}$in (2.33) falls off like $1 / r$ in position space. This naïvely indicates a field strength $F^{-} \sim 1 / r^{2}$ and thus an infrared divergence in the energy after the spatial integration for all $p>1$. However, the actual field strength, $\theta F^{-}=P_{1}$, gives finite energy - it corresponds in position space to a Gaussian falloff. The derivative and commutator terms in the field strength apparently cancel all the power law dependence. In generic commutative theories the $1 / r$ falloff of the gauge field (which is required for an acceptable falloff of the scalar field derivatives) does lead to a divergent energy, and so eliminates the possibility of solitons with more than three transverse directions 1 . So this gives another instance of noncommutative solitons with no commutative counterparts.

\subsection{More on Instantons}

At this point it is natural to ask what class of noncommutative solitons can be generated using our technique. To explain a specific limitation we consider the important example of instantons in a noncommutative $U(1)$ gauge theory in four dimensions [1]. The equations of motion

$$
\left[C^{\mu},\left[C_{\mu}, C_{\nu}\right]\right]=0
$$

where $\mu, \nu=1,2,3,4$ are invariant under $C_{\mu} \rightarrow U C_{\mu} \bar{U}$ for all the $U$ satisfying (2.2), including those of the form (2.4).

1 See e.g. 34. We thank G. Moore for bringing this to our attention. 
Any self-dual or anti-self-dual field strength automatically satisfies (2.39). In the operator formalism the self-duality condition is

$$
\begin{aligned}
{\left[C_{z}, \bar{C}_{\bar{w}}\right] } & =0, \\
{\left[C_{z}, \bar{C}_{\bar{z}}\right] } & =\left[C_{w}, \bar{C}_{\bar{w}}\right],
\end{aligned}
$$

and the anti-self-duality condition reads

$$
\begin{aligned}
{\left[C_{z}, C_{w}\right] } & =0, \\
{\left[C_{z}, \bar{C}_{\bar{z}}\right]+\left[C_{w}, \bar{C}_{\bar{w}}\right] } & =-2 I .
\end{aligned}
$$

We chose complex coordinates in two 2D subspaces with identical noncommutativity, so $\Theta$ is self-dual; furthermore $\epsilon_{z w \overline{z w}}=1$. Now, it is simple to show that the self-duality condition (2.40) is invariant under $U$ transformations satisfying (2.2), including those satisfying (2.4), whereas the anti-self-duality condition (2.41) is invariant only if also $\bar{U} U=I$, i.e. under true gauge transformations. Self-dual instantons are thus simple to generate because the self-duality conditions are preserved under solution generating transformations. Indeed, starting with vacuum and transforming with a suitable $U$ we return to (2.35), recovering the self-dual instantons of [16]. On the other hand, it is more difficult to generate the antiself-dual instantons of Nekrasov and Schwarz [1] since transformations of the vacuum are generally not anti-self-dual. Of course this can also be turned into an advantage: starting with the Nekrasov-Schwarz anti-self-dual instanton we can generate many other solutions that are similarly unconnected to the vacuum.

\section{Applications in String Field Theory}

So far we have discussed noncommutative Yang-Mills-Higgs theories, but it should be clear that our method applies much more generally. The key feature is the existence of $U(\mathcal{H})$ gauge symmetry. In this section we study actions arising from string field theory, and our exact solutions will correspond to D-branes. As discussed in section 4, this generalizes the construction of [8], and shows that the results obtained there are exact for any value of $\theta$.

The first issue we need to discuss is the realization of gauge symmetry in string field theory. Witten's cubic open string field theory [18] is invariant with respect to the gauge transformations

$$
\delta_{\Lambda} \Psi=Q \Lambda+g_{s} \Psi * \Lambda-g_{s} \Lambda * \Psi
$$


where $\Lambda$ is a ghost number zero string field. The $g_{s}$ dependent contribution mixes component fields of different levels. The resulting transformations look very unusual and unwieldy when expressed in component form, and the action truncated to any finite number of components is not gauge invariant. For example, the lowest level component of $\Lambda$ induces the following (schematic) transformations on the tachyon and gauge field [35]:

$$
\begin{aligned}
\delta_{\lambda} \phi= & {[\tilde{\lambda}, \tilde{\phi}]+\{\tilde{\lambda}, \tilde{a}\}+\left\{\partial_{\mu} \tilde{\lambda}, \tilde{A}^{\mu}\right\}+\left\{\tilde{\lambda}, \partial_{\mu} \tilde{A}^{\mu}\right\}+\cdots } \\
\delta_{\lambda} A_{\mu}= & \partial_{\mu} \lambda+\left[\tilde{\lambda}, \tilde{A}_{\mu}\right]+\left[\tilde{a}, \partial_{\mu} \tilde{\lambda}\right]+\left[\tilde{\lambda}, \partial_{\mu} \tilde{a}\right]+\left\{\tilde{\lambda}, \partial_{\mu} \tilde{\phi}\right\}+\left\{\partial_{\mu} \tilde{\lambda}, \tilde{\phi}\right\} \\
& +\left[\partial_{\mu} \tilde{\lambda}, \partial^{\nu} \tilde{A}_{\nu}\right]+\left[\tilde{\lambda}, \partial_{\mu} \partial^{\nu} \tilde{A}_{\nu}\right]+\left[\partial^{\nu} \partial_{\mu} \tilde{\lambda}, \tilde{A}\right]+\left[\partial^{\nu} \tilde{\lambda}, \partial_{\mu} \tilde{A}_{\nu}\right]+\cdots .
\end{aligned}
$$

Here $a$ is an auxilliary scalar, $\cdots$ indicate contributions of higher level fields, and $\tilde{f}=$ $\exp \left[\alpha^{\prime} \ln (3 \sqrt{3} / 4) \partial_{\mu} \partial^{\mu}\right] f$ for any function $f$. Also, Chan-Paton factors have been included and so all fields are $N \times N$ matrices.

To obtain a simpler realization of the gauge invariance we can imagine integrating out classically all fields except for the tachyon and gauge field. Then it is believed [36] that there exists a field redefinition such that

$$
\begin{aligned}
\delta_{\lambda} \phi & =-i[\lambda, \phi], \\
\delta_{\lambda} A_{\mu} & =\partial_{\mu} \lambda-i\left[\lambda, A_{\mu}\right] .
\end{aligned}
$$

In particular, in the presence of noncommutativity there will be a $U(\mathcal{H})$ gauge symmetry with fields transforming as in the examples of the previous section. It is then simple to apply our solution generating technique to arbitrary gauge invariant actions expressed in terms of these variables. This is the approach we follow in this section.

As is well known from the work of [4,5], the B-field is incorporated into the action by replacing ordinary products by $\star$ products, and the closed string metric $g_{\mu \nu}$ and coupling $g_{s}$ by the open string metric $G_{\mu \nu}$ and coupling $G_{s}$. There is some freedom in the choice of the open string quantities corresponding to a choice of $\Phi$ parameter; see [14] for detailed discussion. For our purposes, it is convenient to take $\Phi=-B$. In the case of maximal rank B-field and Euclidean signature this implies the relations

$$
\begin{aligned}
\Theta & =\frac{1}{B}, \\
G & =-\left(2 \pi \alpha^{\prime}\right)^{2} B \frac{1}{g} B, \\
G_{s} & =g_{s} \operatorname{det}\left(2 \pi \alpha^{\prime} B g^{-1}\right)^{\frac{1}{2}} .
\end{aligned}
$$


The noncommutative field strength $F$ only appears in the action in the combination $F+\Phi$, or equivalently given our choice for $\Phi$, only through $[C, \bar{C}]$.

On the other hand, for constructing codimension $2 p$ solutions it is only necessary to turn on noncommutativity in $2 p$ directions. For simplicity we will explicitly consider $p=1$, but the generalization to arbitrary $p$ is straightforward. In $d+1$ Minkowskian dimensions we take $g_{\mu \nu}=\eta_{\mu \nu}$ and

$$
B_{d-1, d}=b, \quad b<0,
$$

so that

$$
\begin{aligned}
\theta & \equiv \Theta^{d-1, d}=\frac{1}{|b|}, \\
G_{\mu \nu} & =\operatorname{diag}\left(1,-1, \ldots,-1,-\left(2 \pi \alpha^{\prime} b\right)^{2},-\left(2 \pi \alpha^{\prime} b\right)^{2}\right), \\
G_{s} & =\left(2 \pi \alpha^{\prime}|b|\right) g_{s} .
\end{aligned}
$$

It is convenient to use complex coordinates in the noncommutative directions,

$$
z=\frac{1}{\sqrt{2}}\left(x^{d-1}+i x^{d}\right) .
$$

In the operator representation we introduce $C$ and $\bar{C}$ as in section 2.

\subsection{Bosonic string}

The action for the tachyon and gauge field in the absence of the B-field is

$$
S=\frac{c}{g_{s}} \int d^{26} x \sqrt{g}\left\{-\frac{1}{4} h(\phi-1) F^{\mu \nu} F_{\mu \nu}+\cdots+\frac{1}{2} f(\phi-1) \partial^{\mu} \phi \partial_{\mu} \phi+\cdots-V(\phi-1)\right\} .
$$

Omitted terms indicate higher powers of fields and higher derivatives. It is convenient for us to take the arguments of $h, f$ and $V$ to be $\phi-1$. We have defined $\phi$ so that $V(\phi-1)$ has a local maximum at $\phi=0$ with $V(-1)=1$ (the $D 25$-brane), and a local minimum at $\phi=1$ with $V(0)=0$ (the closed string vacuum). With these conventions, the $D 25$-brane tension in the absence of B-field is $T_{25}=c / g_{s}$. The functions $h(\phi-1)$ multiplying powers of $F_{\mu \nu}$ without derivatives are all known since they sum up to give $V(\phi-1)$ times the Born-Infeld action. In particular, these functions all vanish in the closed string vacuum [37,38,21]; as will see, this will be important when we come to verifying that our solitons have the correct tensions to be identified with D-branes.

Now we turn on the B-field in $x^{24}, x^{25}$. The tachyon transforms in the adjoint of noncommutative $\mathrm{U}(1)$ as in (2.15), and the field strength becomes

$$
F_{\mu \nu}=\partial_{\mu} A_{\nu}-\partial_{\nu} A_{\mu}+i\left[A_{\mu}, A_{\nu}\right] .
$$


Using the operator representation in the noncommutative directions, the action becomes,

$$
\begin{aligned}
S=\frac{2 \pi \theta c}{G_{s}} \int d^{24} x \sqrt{G} \operatorname{Tr}\{ & -\frac{1}{4} h(\phi-1)\left(F^{\mu \nu}+\Phi^{\mu \nu}\right)\left(F_{\mu \nu}+\Phi_{\mu \nu}\right)+\cdots \\
& \left.+\frac{1}{2} f(\phi-1) D^{\mu} \phi D_{\mu} \phi+\cdots-V(\phi-1)\right\},
\end{aligned}
$$

with

$$
F_{24,25}+\Phi_{24,25}=-i F_{z \bar{z}}+\frac{1}{\theta}=-\frac{1}{\theta}[C, \bar{C}] .
$$

All indices are contracted with $G_{\mu \nu}$, and $\star$ products are implied. Also, we have not specified the factor ordering in (3.10) since it will not be necessary to do so.

Now we repeat the argument from section 2. Let $U \in U(\mathcal{H})$ satisfy $\bar{U} U=I$ and be independent of $x^{0}, \cdots, x^{23}$. The equations of motion following from (3.10) are invariant under the transformations

$$
\begin{aligned}
\phi & \rightarrow U \phi \bar{U}, \\
C & \rightarrow U C \bar{U} \\
A_{\mu} & \rightarrow U A_{\mu} \bar{U}, \quad \mu=0 \ldots 23 .
\end{aligned}
$$

Taking $U=S^{n}$ so that

$$
U \bar{U}=I-P_{n}
$$

and acting on the closed string vacuum - $\phi=1, C=\bar{a}, A_{\mu}=0$ - we thus generate the solutions

$$
\begin{aligned}
\phi & =S^{n} \bar{S}^{n}=\left(I-P_{n}\right), \\
C & =S^{n} \bar{a}^{n}, \\
A_{\mu} & =0, \quad \mu=0 \ldots 23 .
\end{aligned}
$$

They are interpreted as $n$ coincident D23-branes.

It is easy to evaluate the energy of this solution for the general action (3.10) because the only contribution comes from the potential term. In particular, all terms involving covariant derivatives of $\phi$ or $F_{\mu \nu}$ vanish because they do so in the closed string vacuum and this is preserved by the solution generating transformation. We also have

$$
h(\phi-1)[C, \bar{C}]^{2}=h\left(-P_{n}\right)\left(1-P_{n}\right)=h(-1) P_{n}\left(1-P_{n}\right)=0 .
$$

In the second step we used $h(0)=0$. Terms with higher powers of $[C, \bar{C}]$ similarly vanish (with any choice of factor ordering) simply because the projection operators $P_{n}$ and $1-P_{n}$ are orthogonal. For the potential term we have

$$
V(\phi-1)=V\left(-P_{n}\right)=V(-1) P_{n}=P_{n} .
$$


Then using

$$
\frac{\sqrt{G} \theta}{G_{s}}=\frac{2 \pi \alpha^{\prime}}{g_{s}}
$$

we find for the action (3.10) evaluated on our solution,

$$
S=\frac{(2 \pi)^{2} \alpha^{\prime} n c}{g_{s}} \int d^{24} x
$$

which identifies the tension as

$$
T=\frac{(2 \pi)^{2} \alpha^{\prime} n c}{g_{s}}=(2 \pi)^{2} \alpha^{\prime} n T_{25}=n T_{23}
$$

as desired.

Repeating this construction for $2 p$ noncommutative directions we find $D(25-2 p)$ branes with the correct tension.

The construction we have given here reduces to that of [8] in the limit of large B-field. There, the effect of the large B-field was to suppress the derivative contributions. Here, we have an exact construction for any value of $\mathrm{B}$. The key point is that the solution generating transformation generates a gauge field which sets the covariant derivatives of fields to zero.

\subsection{The Superstring}

In type II string theory there are two types of unstable Dp-branes: "wrong" $p$ nonBPS $D p$-branes, and the $D \bar{D}$ system. The construction of solitons on the former class of D-branes is a trivial extension of our discussion for the bosonic string, so we focus here on the $D \bar{D}$ system. The field content of the effective theory after integrating out massive modes corresponds to the theory studied in section 2.3. The tachyon potential is a "Mexican hat" with minima at $|\phi|=1$. There is also a symmetry given by the action of $(-)^{F_{L}}$ which corresponds to interchanging the $D$ and $\bar{D}$ 39:

$$
(-1)^{F_{L}}: \quad \phi \leftrightarrow \bar{\phi}, \quad A^{+} \leftrightarrow A^{-}
$$

Next we write down an action for the string field theory, generalizing (2.22) and satisfying some basic properties. First consider the gauge kinetic terms. Given the symmetry (3.20) we write

$$
\mathcal{L}_{\text {gauge }}=h_{+}\left(|\phi|^{2}-1\right)\left(F_{\mu \nu}^{-}+F_{\mu \nu}^{+}\right)^{2}+h_{-}\left(|\phi|^{2}-1\right)\left(F_{\mu \nu}^{-}-F_{\mu \nu}^{+}\right)^{2}
$$

The function $h_{+}$is known to vanish in the closed string vacuum. 
In the noncommutative theory $F^{-}$and $F^{+}$transform differently under the two $U(1)$ factors, but one can form linear combinations by noting that $F^{-}$and $\bar{\phi} F^{+} \phi$ transform in the same way. So an acceptable gauge kinetic term is

$$
\begin{aligned}
\mathcal{L}_{\text {gauge }} & =h_{+}(\bar{\phi} \phi-1)\left\{F_{\mu \nu}^{-}+\Phi_{\mu \nu}+\bar{\phi}\left(F_{\mu \nu}^{+}+\Phi_{\mu \nu}\right) \phi\right\}^{2} \\
& +h_{-}(\bar{\phi} \phi-1)\left\{F_{\mu \nu}^{-}+\Phi_{\mu \nu}-\bar{\phi}\left(F_{\mu \nu}^{+}+\Phi_{\mu \nu}\right) \phi\right\}^{2} \\
& +\left\{\phi \leftrightarrow \bar{\phi}, \quad A^{+} \leftrightarrow A^{-}\right\},
\end{aligned}
$$

with $h_{+}(0)=0$. The last line is included for symmetry under $(-)^{F_{L}}$. A similar expression holds for terms including higher powers of the gauge fields.

Tachyon kinetic terms appear as in (2.22), but now multiplied by functions $f(\bar{\phi} \phi-1)$ and symmetrized. Symmetry under $(-)^{F_{L}}$ implies that the potential is of the form

$$
V(\phi \bar{\phi}-1)+V(\bar{\phi} \phi-1) \text {. }
$$

In the notation of (2.22) we take $V=W$.

Now we use our solution generating transformation to construct exact solitons representing BPS D-branes. As before, we will explicitly consider the codimension two case; starting with a spacefilling $D 9-\overline{D 9}$ system of IIB this will produce BPS $D 7$-branes. As in (2.25), the solution we generate starting from the closed string vacuum is

$$
\begin{aligned}
\phi & =S^{m} \bar{S}^{n}, \\
C^{-} & =S^{n} \bar{a}^{n}, \\
C^{+} & =S^{m} \bar{a} \bar{S}^{m}, \\
A_{\mu}^{+} & =A_{\mu}^{-}=0, \quad \mu=0 \ldots 7 .
\end{aligned}
$$

We claim that this solution represents $m$ D7-branes coincident with $n \overline{D 7}$-branes.

We now work out the energy of this solution. As in the bosonic case, covariant derivatives of the tachyon and field strengths vanish before and after the transformation, and so do not contribute to the energy. It is less trivial to verify that the gauge field terms (3.22) do not contribute. We need to compute

$$
h_{+}(\bar{\phi} \phi-1)\left\{\left[C^{-}, \overline{C^{-}}\right]+\bar{\phi}\left[C^{+}, \overline{C^{+}}\right] \phi\right\}^{2}+h_{-}(\bar{\phi} \phi-1)\left\{\left[C^{-}, \overline{C^{-}}\right]-\bar{\phi}\left[C^{+}, \overline{C^{+}}\right] \phi\right\}^{2} .
$$

For our solution,

$$
\begin{aligned}
\phi \bar{\phi} & =I-P_{m}, \\
\bar{\phi} \phi & =I-P_{n}, \\
{\left[C^{-}, \overline{C^{-}}\right] } & =-\left(I-P_{n}\right), \\
\bar{\phi}\left[C^{+}, \overline{C^{+}}\right] \phi & =-\left(I-P_{n}\right) .
\end{aligned}
$$


The first term in (3.25) vanishes since $h_{+}(\bar{\phi} \phi-1)=h_{+}\left(-P_{n}\right)=h_{+}(-1) P_{n}$, which is orthogonal to $I-P_{n}$; here we used that $h_{+}(0)=0$. The second term in (3.25) vanishes without a similar assumption about $h_{-}$. So as in the bosonic theory, the only contribution to the energy comes from the potential term, which we find to be

$$
V(\phi \bar{\phi}-1)+V(\bar{\phi} \phi-1)=V\left(-P_{m}\right)+V\left(-P_{n}\right)=V(-1)\left(P_{m}+P_{n}\right)
$$

Repeating the computation leading to (3.19) in the bosonic case now gives the tension

$$
T_{n m}=\frac{(2 \pi)^{2} \alpha^{\prime}(n+m) c}{g_{s}} T_{9}=(n+m) T_{7} .
$$

as expected for $m D 7$-branes plus $n \overline{D 7}$-branes.

Using the ABS construction of solitons in section 2.4, it is straightforward to generalize the above discussion to codimension $2 p$ solitons representing coincident $D(9-2 p)$ and $\overline{D(9-2 p)}$ branes.

\section{Discussion}

In this paper we have constructed soliton solutions of noncommutative gauge theories with or without scalar (tachyon) fields for arbitrary values of the noncommutativity parameter $\theta$. In the context of an effective low-energy description of string field theory these solutions represent various D-brane solutions discussed previously at infinite noncommutativity in [7,8,9]. We would now like to discuss some of the implications of these solutions and the technique used to generate them.

One issue which has arisen in discussions of noncommutative tachyon condensation involves the correct description of the closed string vacuum. Clearly in the vacuum the tachyon field $\phi$ takes on its minimum everywhere, $\phi=\phi_{*} I$, but various possibilities have been discussed for the gauge field configuration. Given the vanishing of the gauge kinetic term in the closed string vacuum, these different choices are degenerate in energy at the level of effective field theory. It was argued in [40], based in part on studies in truncated string field theory [41], that these choices are in fact illusory, an artifact of using the wrong variables to describe the closed string vacuum.

In [8,9] one obtains a simple description of $D$-branes as noncommutative solitons by taking a limit of large noncommutativity, the key point being that derivative corrections are suppressed in this limit. In [8] it was suggested that this description should also hold 
at finite or vanishing noncommutativity since a purely transverse B-field can be shifted by a gauge transformation, but it was not clear how to show this explicitly given the presence of derivative corrections. Since the limit also requires one to take the closed string coupling $g_{s} \rightarrow 0$ in order to obtain a finite open string coupling, another concern was whether this limit of the theory is smoothly connected to the commutative theory with finite but small $g_{s} .2$ Various discussion of these issues can be found in [43, 14, 40, 44,

To resolve these issues one should distinguish several possible notions of " $\theta$ " independence. In string theory the gauge invariant physical quantity is $\mathcal{B} \mathcal{F}=F+B$. Different choices of $F$ and $B$ with fixed $\mathcal{F}$ lead to different but gauge equivalent descriptions of the physics. The physical issue is one of the $\mathcal{F}$ dependence of the D-brane tensions and spectra computed using noncommutative solitons.

The noncommutative description of D-branes involves the noncommutative field strength $\hat{F}$ and a two-form $\Phi$ [45,5, 14]. In this language the issue is one of dependence on the physical combination $\hat{F}+\Phi$. The discussion of [14] makes it clear that one can formulate the theory in a form which is independent of the background value of this physical combination. In this language one must choose a classical solution for the quantities $X^{i}=\Theta^{i j} C_{j}$ with specified boundary conditions at infinity. Specifying boundary conditions is equivalent to choosing a vacuum, $X^{i}=x^{i}$ with $\left[x^{i}, x^{j}\right]=i \Theta^{i j}$, and the question of $\theta$ independence is whether the physics is independent of this choice. Both [8] and [43] were based on the vacuum $X^{i}=0$ : [ 8 phrased this in terms of the limit $B \rightarrow \infty$ with $\Theta=1 / B$, while 43] set $C_{i}=0$. 4 Here we have shown that the correct properties of D-branes are recovered for any choice of vacuum, that is

$$
X^{i}=\left(\frac{1}{B}\right)^{i j} C_{j}, \quad \text { with } \frac{1}{\sqrt{2}}\left(C_{2 \alpha-1}-i C_{2 \alpha}\right)=\bar{a}_{\alpha},
$$

for any $B$. Since D-brane properties are here found to be $\theta$ independent, this result also supports the argument of 40] that the superficially different vacua labelled by $\theta$ should actually be identified.

Since we can in particular take $\theta$ finite, we are not forced to take $g_{s} \rightarrow 0$ in order to make the open string coupling finite. Thus the representation of D-branes as non-

2 Similar issues arise in the proposal of 42 .

3 Here $F$ is the commutative field strength. The noncommutative field strength denoted $F$ in the main text is changed to $\hat{F}$ in the discussion section, conforming with the notation of [5].

4 More precisely, [8] took $B \rightarrow \infty$ in transverse directions, while [43] took $C_{i}=0$ in all directions including time. 
commutative solitons we have found is valid for arbitrary $\theta$ and small but non-zero $g_{s}$. This establishes that the solutions found in [8,9] are not just a description of D-branes in some unphysical regime of infinite noncommutativity and zero coupling, but rather are describing D-branes in the conventional weakly coupled string theory vacuum as argued in [8].

Another issue raised by this work is related to the presence of non-zero gauge fields in the exact solution, even in the bosonic string. This would naïvely seem to be in contradiction with the description of D-branes as lumps in open bosonic string field theory [23] where one argues that the gauge field can consistently be set to zero in level truncation [35,46. On the other hand, the relation between the gauge invariant description used here and the gauge fields which appear in string field theory is highly non-trivial [36]. It may be that a field redefinition and/or string field theory gauge transformation relates the two naïvely different descriptions. In type II string theory the question can be asked more sharply since the gauge field strength acts as a source of RR charge and hence must be non-zero in any construction of BPS D-branes. To our knowledge the form of the gauge field for BPS D-branes has not yet been examined in truncated open string field theory.

Finally, it would be interesting to apply these techniques directly in string field theory. Cubic open string field theory [18] even at $B=0$ has an infinite dimensional symmetry group, and it may be possible to construct the analog of non-unitary isometries. It has been suggested [27] that one might construct the closed string vacuum in the form

$$
A_{0}=\bar{V} * Q V \text {, }
$$

for some "non-unitary isometry" obeying $\bar{V} * V=I$. 5 Our considerations suggest that whether or not this is the case, one should try to construct D-brane solutions in string field theory by acting with non-unitary isometries on the closed string vacuum state

$$
\left(Q+A_{\mathrm{D}-\text { brane }}\right)=V *\left(Q+A_{0}\right) \bar{V} .
$$

It would be interesting to try to carry out this proposal concretely, either in the theory with $B=0$ or in the theory with $B \neq 0$ where the star product is modified to include the Moyal product on the zero mode wave functions.

Acknowledgements: We thank D. Kutasov, E. Martinec and G. Moore for discussions. This work was supported in part by NSF grant PHY-9901194 and by DOE grant DEFG0290ER-40560. FL was supported in part by a Robert R. McCormick fellowship.

5 This proposal was also made by G. Moore at Aspen in August, 2000. 


\section{References}

[1] N. Nekrasov and A. Schwarz, "Instantons on noncommutative $R^{4}$ and $(2,0)$ superconformal six dimensional theory", Commun. Math. Phys. 198 (1998) 689, hepth/9802068.

[2] A. Connes, M. Douglas and A. Schwarz, "Noncommutative Geometry and Matrix Theory:Compactification on Tori," JHEP 02 (1998) 003; hep-th/9711162.

[3] M. R. Douglas and C. Hull, "D-branes and the Noncommutative Torus," JHEP 9802 (1998) 008:hep-th/9711165.

[4] V. Schomerus, "D-Branes and Deformation Quantization", JHEP 9906 (1999) 030, hep-th/9903205.

[5] N. Seiberg and E. Witten, "String theory and noncommutative geometry," JHEP 9909, 032 (1999), hep-th/9908142.

[6] R. Gopakumar, S. Minwalla and A. Strominger, "Noncommutative solitons", JHEP 0005 (2000) 020 , hep-th/0003160.

[7] K. Dasgupta, S. Mukhi and G. Rajesh, "Noncommutative tachyons," JHEP 0006, 022 (2000) hep-th/0005006.

[8] J. A. Harvey, P. Kraus, F. Larsen and E. J. Martinec, "D-branes and strings as noncommutative solitons", JHEP 0007 (2000) 042, hep-th/0005031.

[9] E. Witten, "Noncommutative tachyons and string field theory", hep-th/0006071.

[10] D. J. Gross and N.A.Nekrasov, "Monopoles and Strings in Noncommutative Gauge Theory," JHEP 0007 (2000) 034; hep-th/ 0005204; D. J. Gross and N. A. Nekrasov, "Dynamics of Strings in Noncommutative Gauge Theory," hep-th/0007204.

[11] A. P. Polychronakos, "Flux tube solutions in noncommutative gauge theories," hepth/0007043.

[12] D. P. Jatkar, G. Mandal and S. R. Wadia, "Nielsen-Olesen vortices in noncommutative Abelian Higgs model", JHEP 0009 (2000) 018 , hep-th/0007078.

[13] C. Sochichiu, "Noncommutative Tachyonic Solitons. Interaction with Gauge Field," hep-th/0007217.

[14] N. Seiberg, "A Note on Background Independence in Noncommutative Gauge Theories, Matrix Model and Tachyon Condensation," hep-th/0008013.

[15] D. Bak, "Exact multi-vortex solutions in noncommutative Abelian-Higgs theory", hep-th/0008204.

[16] M. Aganagic, R. Gopakumar, S. Minwalla and A. Strominger, "Unstable solitons in noncommutative gauge theory", hep-th/0009142.

[17] R. Tatar, "A Note on Non-Commutative Field Theory and Stability of BraneAntibrane Systems," hep-th/0009213.

[18] E. Witten, "Noncommutative Geometry And String Field Theory", Nucl. Phys. B268 (1986) 253. 
[19] E. Witten, "On background independent open string field theory", Phys. Rev. D46 (1992) 5467, hep-th/9208027.

[20] A. A. Gerasimov and S. L. Shatashvili, "On exact tachyon potential in open string field theory", hep-th/0009103.

[21] D. Kutasov, M. Marino and G. Moore, "Some exact results on tachyon condensation in string field theory", hep-th/0009148.

[22] D. Ghoshal and A. Sen, "Normalisation of the background independent open string field theory action", hep-th/0010021; L. Cornalba, "Tachyon Condensation in Large Magnetic Fields with Background Independent String Field Theory", hep-th/0009191; K. Okuyama, "Noncommutative Tachyon from Background Independent Open String Field Theory", hep-th/0010028.

[23] J. A. Harvey and P. Kraus, "D-branes as unstable lumps in bosonic open string field theory", JHEP 0004 (2000) 012 , hep-th/0002117; N. Berkovits, A. Sen and B. Zwiebach, "Tachyon condensation in superstring field theory", hep-th/0002211; R. de Mello Koch, A. Jevicki, M. Mihailescu and R. Tatar, "Lumps and p-branes in open string field theory", Phys. Lett. B482, 249 (2000), hep-th/0003031; N. Moeller, A. Sen and B. Zwiebach, "D-branes as tachyon lumps in string field theory", JHEP 0008, 039 (2000), hep-th/0005036.

[24] P. Ho, "Twisted bundle on noncommutative space and U(1) instanton", hepth/0003012.

[25] K. Furuuchi, "Equivalence of projections as gauge equivalence on noncommutative space", hep-th/0005199; K. Furuuchi, "Topological Charge of $U(1)$ Instantons on Noncommutative $R^{4 "}$ hep-th/0010006.

[26] N. A. Nekrasov, "Noncommutative Instantons Revisited," hep-th/0010017.

[27] M. Schnabl, "String field theory at large B-field and noncommutative geometry," hepth/0010034.

[28] B. de Wit, J. Hoppe and H. Nicolai, "On the quantum mechanics of supermembranes," Nucl. Phys. B305, 545 (1988).

[29] L. Cornalba, "D-brane physics and noncommutative Yang-Mills theory", hep-th/9909081;

N. Ishibashi, "A relation between commutative and noncommutative descriptions of D-branes", hep-th/9909176.

[30] M. F. Atiyah, R. Bott and A. Shapiro, "Clifford Modules", Topology 3 suppl. 1 (1964) 3.

[31] E. Witten, "D-branes and K-theory", JHEP 9812 (1998) 019, hep-th/9810188; P. Horava, "Type IIA D-branes, K-theory, and matrix theory", Adv. Theor. Math. Phys. 2 (1999) 1373, hep-th/9812135.

[32] J. A. Harvey and G. Moore, "Noncommutative tachyons and K-theory", hepth/0009030. 
[33] U. Venugopalkrishna, "Fredholm operators associated with strongly pseudo convex domains in $C^{n}$," J. Functional Anal. 9 (1972) 349; L. Boutet de Monvel, "On the index of Toeplitz operators of several complex variables," Inv. Math. 50 (1979) 249.

[34] See sec. 3.3 of S.Coleman, "Quantum lumps and their classical descendants," in Aspects of Symmetry, Cambridge University Press, 1985.

[35] V .A. Kostelecky and S.Samuel, "On a Nonperturbative Vacuum for the Open Bosonic String," Nucl.Phys. B336 (1990) 263.

[36] J. R. David, "U(1) gauge invariance from open string field theory," hep-th/0005085.

[37] A. Sen, "Supersymmetric world-volume action for non-BPS D-branes," JHEP 9910, 008 (1999) hep-th/9909062.

[38] J. A. Harvey, D. Kutasov and E. J. Martinec, "On the relevance of tachyons," hepth/0003101.

[39] A. Sen, "BPS D-branes on non-supersymmetric cycles," JHEP 9812 (1998) 021, hepth/9812031.

[40] A. Sen, "Some issues in non-commutative tachyon condensation", hep-th/0009038;

A. Sen, "Uniqueness of tachyonic solitons", hep-th/0009090.

[41] A. Sen and B. Zwiebach, "Large marginal deformations in string field theory," JHEP 0010, 009 (2000) [hep-th/0007153]; W. Taylor, "Mass generation from tachyon condensation for vector fields on D-branes," JHEP 0008, 038 (2000) [hep-th/0008033];

A. Iqbal and A. Naqvi, "On marginal deformations in superstring field theory," hepth/0008127.

[42] E. Witten, "Overview of K Theory Applied to Strings," hep-th/0007175.

[43] R. Gopakumar, S. Minwalla and A. Strominger, "Symmetry restoration and tachyon condensation in open string theory", hep-th/0007226.

[44] P. Kraus, A. Rajaraman and S. Shenker, "Tachyon condensation in noncommutative gauge theory", hep-th/0010016.

[45] B. Pioline and A. Schwarz, "Morita equivalence and T-duality (or B versus Theta)," JHEP 9908 (1999) 021;hep-th/9908019.

[46] A. Sen and B. Zwiebach, "Tachyon condensation in string field theory", JHEP 0003 (2000) 002, hep-th/9912249; N. Moeller and W. Taylor, "Level truncation and the tachyon in open bosonic string field theory", Nucl. Phys. B583 (2000) 105, hepth/0002237. 